\title{
Peri-operative treatment of sleep-disordered breathing and outcomes in bariatric patients
}

\author{
Jacques-Henri Meurgey $^{1}$, Richard Brown ${ }^{2}$, Asia Woroszyl-Chrusciel ${ }^{2}$, Joerg Steier $^{1,2}$ \\ ${ }^{1}$ King's College London, Faculty of Life Sciences and Medicine, London, UK; ${ }^{2}$ Lane Fox Respiratory Unit and Sleep Disorders Centre, Guy's \& St \\ Thomas' NHS Foundation Trust, London, UK \\ Contributions: (I) Conception and design: JH Meurgey, J Steier; (II) Administrative support: J Steier; (III) Provision of study materials or patients: J \\ Steier, R Brown, A Woroszyl-Chrusciel; (IV) Collection and assembly of data: JH Meurgey, R Brown, A Woroszyl-Chrusciel; (V) Data analysis and \\ interpretation: JH Meurgey, J Steier; (VI) Manuscript writing: All authors; (VII) Final approval of manuscript: All authors. \\ Correspondence to: Joerg Steier. Lane Fox Unit, St Thomas' Hospital, Westminster Bridge, SE1 7EH, London, UK. Email: Joerg.steier@kcl.ac.uk.
}

\begin{abstract}
Background: Obstructive sleep apnoea (OSA) is increasingly common in bariatric patients undergoing sedation during elective surgery. However, it has not been established how significant a contributor it is to peri-operative respiratory complications and mortality. We sought to pre-operatively identify OSA in bariatric patients and record peri-operative complications during and after bariatric surgery.

Methods: Data were collected and analysed from June 2014 to March 2017 for 410 bariatric surgery patients referred to the sleep laboratory for pre-operative screening and treatment of OSA. The STOP-Bang questionnaire, Epworth Sleepiness Scale (ESS) and nocturnal pulse oximetry were recorded and treatment was allocated with continuous positive airway pressure (CPAP). Peri-operative complications and mortality were the primary outcome measures for patients receiving CPAP treatment for OSA, with patients who did not require CPAP used as control. The mean follow-up time for all patients was 433 and 732 days for the patients who had undergone bariatric surgery. The two groups were compared with Chi square test and unpaired two-tailed $t$-test.
\end{abstract}

Results: OSA was present in any form in $70 \%$ of the screened patients; $40 \%$ of patients involved in the study received CPAP treatment. Patients receiving CPAP treatment [49.5 (11.3) years old, 61\% female, $\left.50.3(8.5) \mathrm{kg} / \mathrm{m}^{2}\right]$ were older, had a lower percentage of females and had a higher body mass index (BMI) than those not receiving CPAP [44.9 (12.0), 81\% female, 46.6 (7.7)]. No significant differences were observed between patients on CPAP and those not on CPAP, there was no significant difference in hospital stay or rate of respiratory complications. Out of 53 patients who had undergone bariatric surgery at the cut-off date, only 1 had suffered a respiratory complication.

Conclusions: Bariatric patients who are screened pre-operatively for OSA and treated according to guidelines have no increased risk of respiratory complications compared to patients without OSA.

Keywords: Obstructive sleep apnoea (OSA); sleep apnoea; obesity; continuous positive airway pressure (CPAP)

Submitted Sep 20, 2017. Accepted for publication Sep 26, 2017.

doi: $10.21037 /$ jtd.2017.10.11

View this article at: http://dx.doi.org/10.21037/jtd.2017.10.11

\section{Introduction}

The rate of obesity in the UK is one of the highest in the world (1). As of 2013, 24\% of men and $25 \%$ of women in the UK are classified as obese, with $3 \%$ classified as type III obese [previously known as "morbid obesity" with a body mass index (BMI) above $40 \mathrm{~kg} / \mathrm{m}^{2}$ ] (2). Obesity is linked to several respiratory conditions such as pneumonia, asthma, pulmonary embolism and obstructive sleep apnoea (OSA) (3). However, despite the increasing prevalence of obesity, up to $90 \%$ of OSA remains undiagnosed (4): $4 \%$ of middle-aged men and $2 \%$ of middle-aged women in the UK suffer from sleep apnoea (5), but severe OSA occurs in 
$10-20 \%$ of patients with a BMI above $35 \mathrm{~kg} / \mathrm{m}^{2}$ (6). OSA and obesity are significant contributors to cardiovascular and metabolic morbidity and mortality, associated with diabetes mellitus, ischaemic heart disease, hypertension and depression $(5,6)$.

In OSA, transient hypoxias induced by upper airway occlusion in sleep activate the sympathetic nervous system, increase intra-thoracic pressure swings and cause arousal from sleep (5). Heart rate increases and sleep is fragmented, the sleep quality decreases (7), causing excessive daytime sleepiness, as well as impaired cognitive function, eventually leading to an impaired quality of life (5). OSA is diagnosed by experts with the help of a sleep study (polysomnography) in conjunction with a subjective increase in daytime sleepiness, assessed with the aid of an Epworth Sleepiness Scale (ESS) questionnaire (5).

Continuous positive airway pressure (CPAP) is currently the best available treatment for moderate to severe OSA, effective to avoid upper airway collapse, inflate the chest and diminish work for breathing and neural respiratory drive $(5,8)$. In the context of surgery and anaesthetics, obesity and OSA are associated with a greater incidence in complications both peri- and post-operatively, leading to longer in-hospital stays and putting additional strain on healthcare providers (9). Obesity itself is not necessarily linked to increased risk of pulmonary complications pre- or post-operatively, even in the morbidly obese (10), however, in conjunction with OSA this is different (11). Patients with OSA are more than twice as likely to develop post-operative desaturations, cardiac complications, adult respiratory distress syndrome (ARDS), respiratory failure, aspiration pneumonia and other problems that require admission to intensive care $(9,11)$. Frequent and extended desaturations also predispose patients towards opioid-induced respiratory depression due to hypoxaemia and hypercapnia (6). However, the risk of these complications is potentially reduced if OSA is identified pre-operatively and treated with $\operatorname{CPAP}(4,6,9,11)$.

We hypothesize that long-term and peri-operative complication rates are low in bariatric patients who are screened for OSA and, if required, treated accordingly. For this purpose, we identified peri- and post-operative mortality with long-term follow-up, as well as complication rate and length of hospital stay in all screened bariatric patients and compared the occurrence of complications between patients with moderate-severe OSA with those who had mild OSA or no sleep-disordered breathing.

\section{Methods}

Retrospective data of patients referred to the Lane Fox Unit (respiratory high dependency unit, St. Thomas' Hospital, London, UK) and the Sleep Disorders Centre (Guy's Hospital, London, UK) for assessment of sleep-disordered breathing in conjunction with bariatric surgery were analysed. The study was registered as a service review and approved by the local clinical governance board (registration number: GSTT-2017-6952). Cases were included between 12 June 2014 and 9 March 2017.

Variables recorded included age (years), gender (male and female), height (m), weight at time of sleep study (kg), BMI $\left(\mathrm{kg} / \mathrm{m}^{2}\right)$, neck circumference, waist circumference, comorbidities, medication, surgery date, surgery type, intraoperative complications, post-operative complications, length of post-operative hospital stay (days), ward $v s$. ITU stay, post-operative weight at last follow-up $(\mathrm{kg})$, postoperative BMI at last follow-up $\left(\mathrm{kg} / \mathrm{m}^{2}\right)$, weight loss over the follow-up period (kg/year); it was recorded whether patients were prescribed CPAP, the respective CPAP pressure (in $\mathrm{cmH}_{2} \mathrm{O}$ ), annual CPAP compliance (\% of nights used), average nightly CPAP use (in hours), ESS (points) and Hospital Anxiety and Depression Scale (HADS, points), as well as the results of the nocturnal pulse oximetry data.

\section{Questionnaires}

Patients scheduled to undergo bariatric surgery at Guy's and St. Thomas' NHS Foundation Trust, London, UK were screened pre-operatively with the STOP-Bang questionnaire. STOP-Bang is an acronym for snoring (significantly loud), tired (excessive daytime sleepiness), observed apnoea (others seeing the patient choking during sleep), (blood) pressure (current or treated hypertension), BMI $\left(>35 \mathrm{~kg} / \mathrm{m}^{2}\right)$, age $(>50$ years), neck circumference $(>40 \mathrm{~cm})$ and gender (male). Each parameter is graded "0" (for "no" answers) and "1" (for "yes" answers) (12). A score $>4$ represented a risk of OSA and indicated referral to the Sleep Disorders Centre for more extensive investigation.

The ESS was recorded; it asks patients to rate the probability of falling asleep during eight activities of daily living on a scale of " 0 " to " 3 ", with higher scores $(>10)$ indicating daytime sleepiness (13).

The HADS is a scoring tool to assess patient's levels of anxiety and depression. There are 14 statements to score from " 0 " to " 3 ", 7 about anxiety and 7 about depression. The questions deliberately avoid asking about sleep because 
changes in sleep may be symptoms of these disorders (14). This scale can be used in conjunction with the ESS (10). A score $<9 / 21$ on either questionnaire or a total score $<18 / 42$ points is deemed normal.

\section{Nocturnal pulse oximetry}

Patients recorded two nights of pulse oximetry at home using a Pulsox 300i (Konica Minolta sensing Inc., Hachioji, Tokyo, Japan). The data recorded and calculated included:

(I) Mean pulse rate $\left(\mathrm{min}^{-1}\right)$;

(II) Number of pulse rises $>$ six beats per minute ("pulse rises") $\left(h^{-1}\right)$;

(III) Mean peripheral capillary oxygen saturation $\left(\mathrm{SpO}_{2}, \%\right)$;

(IV) Nadir peripheral capillary oxygen saturation $\left(\mathrm{SpO}_{2}, \%\right)$;

(V) $4 \%$ and $3 \%$ oxygen desaturation index $\left(\mathrm{ODI} ; \mathrm{h}^{-1}\right)$;

(VI) Time spent with $\mathrm{SpO}_{2}<90 \%$ (\% of total time recorded).

The results were further assessed by an expert sleep technician and scored by a consultant. OSA was defined by the number of desaturations a patient experienced per hour of sleep; the $4 \%$ ODI. A $4 \%$ ODI $<5$ events/hour was considered normal; 5-15 events/hour was mild, 15-30 events events/hour was moderate and $>30$ events/hour was considered severe OSA (9). Possible coexisting obesity hypoventilation syndrome (OHS) was indicated by prolonged periods ( $>10 \%$ of the recorded time) of hypoxia $\left(\mathrm{SpO}_{2}<90 \%\right)$ during sleep. Patients were assigned a value from $1-5$ for the severity of their OSA:

(I) "1"-if no OSA present;

(II) "2"-if mild OSA present;

(III) " 3 "-if moderate OSA present;

(IV) "4"-if severe OSA present;

(V) "5"-if patients had OSA, potentially combined with OHS.

Recommended treatments were assigned a value from 1-5:

(I) "1"-no further action necessary;

(II) " 2 " - recommendation of a mandibular advancement device if symptomatic;

(III) "3"- -recommended extubation onto CPAP;

(IV) “4”-peri-operative CPAP;

(V) "5"-referral for inpatient assessment to investigate the potential presence of OHS.

\section{Statistical analysis}

Statistical analysis was performed using the Statistical Package for the Social Sciences (SPSS, IBM, Chicago, IL, USA) version 24.0. Categorical variables are summarised using percentages. A Shapiro-Wilk with Lilliefors significance correction test was used to determine whether continuous variables were normally or non-normally distributed. Normally-distributed continuous variables are cited as "mean (standard deviation)" and compared using an unpaired $t$-test. Non-normally distributed continuous variables are cited as "median [interquartile range (IQR)]" and compared using a Mann-Whitney test. Categorical variables were compared with a Chi-square test. A Pearson correlation test was used to compare independent and dependent variables; a Spearman correlation test was used to compare non-normally distributed data. A P value of $<0.05$ was deemed statistically significant.

\section{Results}

\section{Demographics}

A total of 410 patients awaiting bariatric surgery were studied, the majority were female, middle aged and obese [74\% female (302:108), age 46.9 (11.9) years, weight $134.5(27.5) \mathrm{kg}$, BMI: $48.1(8.2) \mathrm{kg} / \mathrm{m}^{2}$, waist circumference of $136.7(16.7) \mathrm{cm}$, neck circumference $43.6(4.5) \mathrm{cm}]$. Four patients were excluded from the analysis because they had insufficient or unobtainable sleep results. The average of patients was not excessively sleepy, but were considerably susceptible to depression and/or anxiety [ESS 8.0 (5.0) points, total HADS 17.7 (7.3) points]. Most patients $(85.9 \%, \mathrm{n}=352)$ had a BMI $>40 \mathrm{~kg} / \mathrm{m}^{2}, 12.4 \%(\mathrm{n}=51) \mathrm{had}$ a BMI between $35-40 \mathrm{~kg} / \mathrm{m}^{2}$ and $1.7 \%(\mathrm{n}=7)$ patients had a BMI between $30-35 ; 34.2 \%(n=140)$ of the patients were "super obese" with a BMI $\geq 50 \mathrm{~kg} / \mathrm{m}^{2}$; the highest BMI was $88.9 \mathrm{~kg} / \mathrm{m}^{2}$.

\section{Sleep disordered breathing (SDB)}

Nocturnal pulse oximetry data revealed mild-moderate OSA for the total cohort [4\% ODI of $15.4(17.3) \mathrm{h}^{-1}, 3 \%$ ODI of $20.1(19.6) \mathrm{h}^{-1}$, mean $\mathrm{SpO}_{2}$ of 94.1 (3.6) \%, a nadir $\mathrm{SpO}_{2}$ of $75.9(10.0) \%$, mean pulse rise index of 34.0 (18.6) $\mathrm{h}^{-1}$, and a time below $\mathrm{SpO}_{2}$ of $90 \%$ of 8.0 (14.2) \% of the sleep study)]; 285 out of 410 patients (70\%) had at least some 
Table 1 Comparison of male and female cohort

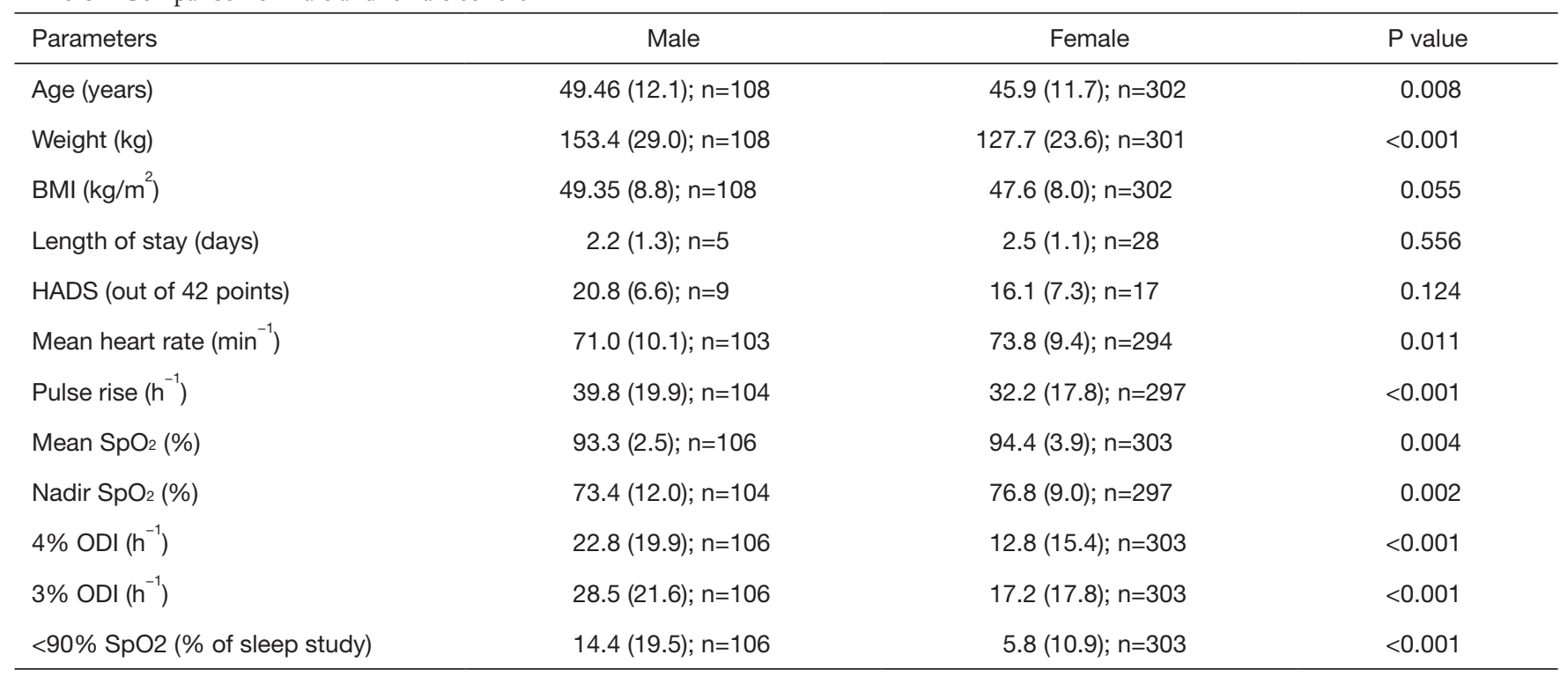

BMI, body mass index; ODI, oxygen desaturation index; OSA, obstructive sleep apnoea; HADS, Hospital Anxiety and Depression Scale.

degree of OSA, while 125 (30\%) had no significant degree of OSA. Of those with any degree of OSA, 127 (31\% of total patients) were mild, 82 (20\% of total patients) were moderate, 57 (14\% of total patients) had severe OSA and $19(5 \%)$ had OSA with potentially combined OHS. Average follow-up time post-pulse oximetry was 432.9 (241.9) days.

\section{Male vs. female groups}

When comparing male with female patients, the male group was slightly older but of similar BMI, both groups had a similar sleep and mental health symptom score (ESS, HADS) and heart rate; there were about three times as many women as men in this cohort. There was no significant difference in length of hospital stay, with all but two patients admitted to a ward post-operatively; the two patients admitted to ICU were female. Men were on average heavier and had more severe sleep-disordered breathing, as indicated by the mean/minimum $\mathrm{SpO}_{2}$, the ODI and the percentage of the night with an $\mathrm{SpO}_{2}<90 \%$, as well as the pulse rise index as an indicator of arousals; men were $20.3 \%$ more likely ( $85 \%$ of male patients compared to $64.7 \%$ of female patients) to require treatment recommendation like $\mathrm{MAD}$, CPAP or referral for further respiratory assessment (Table 1).

\section{Co-morbidities}

Hypertension was present in $42 \%(\mathrm{n}=171)$ patients, $37 \%$ $(\mathrm{n}=153)$ patients had diabetes mellitus, 25\% $(\mathrm{n}=104)$ had depression, 9\% ( $n=37)$ had asthma, 7\% (n=30) had high cholesterol/dyslipidaemia, $7 \%(\mathrm{n}=28)$ had polycystic ovarian syndrome (PCOS), 5\% ( $\mathrm{n}=19)$ had impaired glucose tolerance, 5\% ( $\mathrm{n}=19)$ had anxiety, 3\% ( $\mathrm{n}=13)$ had chronic obstructive pulmonary disease (COPD), $2 \%(n=9)$ had chronic kidney disease (CKD)/renal impairment, 2\% ( $\mathrm{n}=8)$ had epilepsy or a history of seizures.

\section{Medication}

Twenty-five percent ( $\mathrm{n}=102)$ were taking one (or more) oral hyperglycaemic agent(s) (metformin, sitagliptin, gliclazide, liraglutide), $21 \%(\mathrm{n}=87)$ were taking a statin (simvastatin, atorvastatin, rosuvastatin), $17 \%(\mathrm{n}=69)$ were taking one (or more) antidepressant(s) (escitalopram/citalopram, fluoxetine/duloxetine/paroxetine/sertraline, amitriptyline), $17 \%(\mathrm{n}=68)$ were taking a proton-pump inhibitor (omeprazole, lansoprazole), 16\% $(\mathrm{n}=64)$ were taking a calcium channel blocker (amlodipine, nifedipine, diltiazem), $15 \%(\mathrm{n}=61)$ were taking an angiotensin-converting enzyme inhibitor (“ACEi”; ramipril, lisinopril, perindopril, 
Table 2 Comparison of patients with CPAP vs. those without CPAP

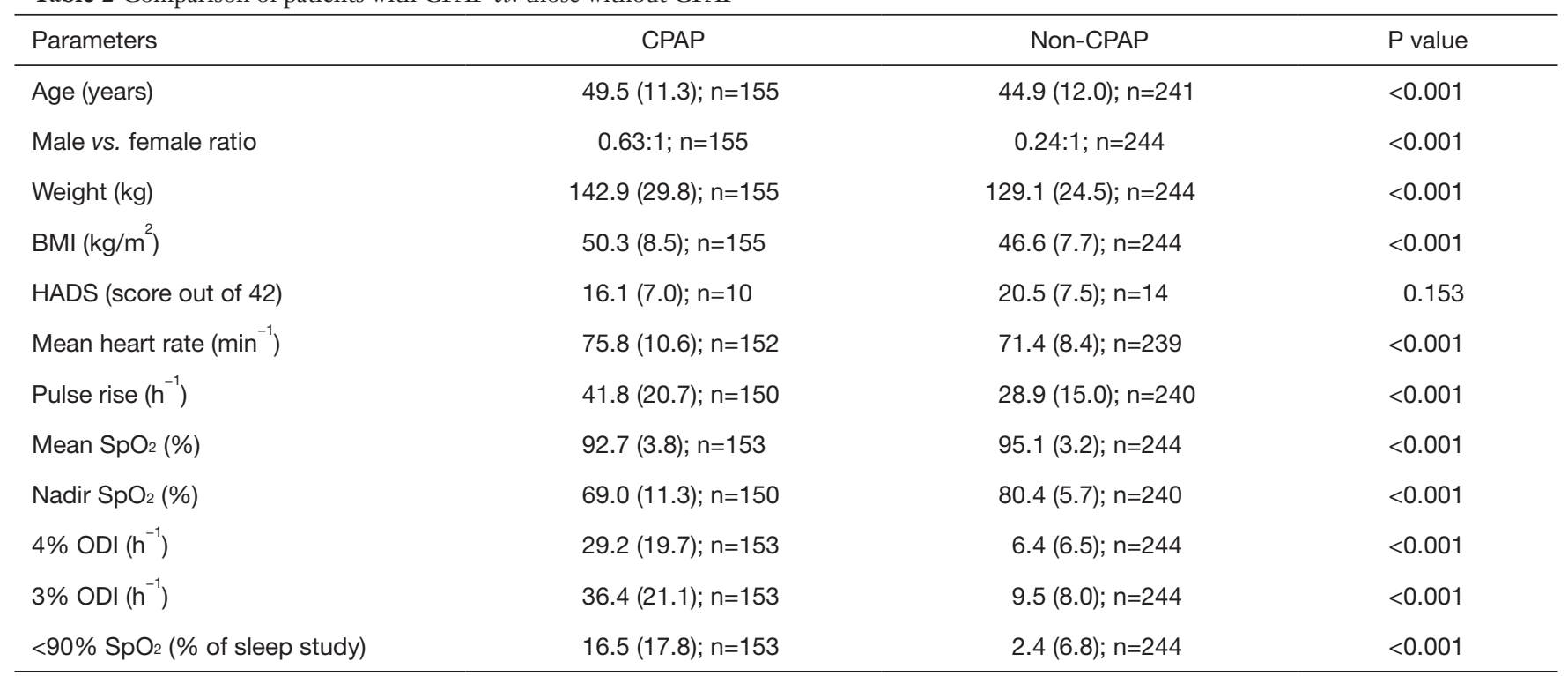

LOS, length of stay; BMI, body mass index; ESS, Epworth Sleepiness Scale; HADS, Hospital Anxiety and Depression scale; ODI, oxygen desaturation index.

enalapril), $14 \%(\mathrm{n}=59)$ were taking one (or more) inhaler(s) (salbutamol, salmeterol, seretide, ventolin, tiotropium, symbicort, clenil modulite, beclomethasone, fluticasone), $9 \%(\mathrm{n}=37)$ were taking one (or more) injected insulin(s) (Humulin, Humalog, "insulin", novomix, Novorapid), $9 \%(\mathrm{n}=37)$ were taking an angiotensin II receptor blocker ("ARB"; losartan, irbesartan), 6\% ( $\mathrm{n}=25)$ were taking one (or more) anti-convulsant (gabapentin, pregabalin, carbamazepine, valproate), $6 \%(\mathrm{n}=25)$ were taking orlistat.

\section{CPAP treatment}

A total of 220 (54\%) patients received no further treatment, $164(40 \%)$ patients were prescribed CPAP treatment and $26(6 \%)$ patients were recommended a mandibular advancement device for their symptoms. Patients who required CPAP therapy were, on average, older, heavier, with a higher BMI and more symptomatic than patients without, as via selection, they had a more severe degree of OSA (Table 2). Of those on CPAP ( $\mathrm{n}=164), 125$ (76\%) were prescribed CPAP pre- and post-operatively, 17 (10\%) were prescribed post-operative extubation onto CPAP, and $22(13 \%)$ were referred to the Lane Fox Unit for further respiratory assessment of non-invasive ventilation (NIV) $v s$. CPAP; 2 of the patients referred for further assessment did not require CPAP. The patients with CPAP had an average pressure of $13.7(2 ; \mathrm{n}=81) \mathrm{cmH}_{2} \mathrm{O}$, an annual compliance of $64.0 \%(34.0 ; n=50)$, and nightly use of $4.5(1.9 ; n=50)$ hours. The length of stay in hospital for the operated cohort was similar between CPAP and non-CPAP group.

\section{Bariatric surgery}

During the follow-up period (i.e., days since sleep study recording) of $719.4(143 ; \mathrm{n}=53)$ days 53/413 (12.8\%) patients had undergone bariatric surgery [ $80 \%$ female (44:9), age $48.5(11.4)$ years, $\left.138.0(28.5) \mathrm{kg}, 49.3(8.2) \mathrm{kg} / \mathrm{m}^{2}\right]$. 48 patients were class III obese, including 20 patients who were "super obese", and 5 patients were class I and II obese. 47\% ( $\mathrm{n}=25)$ patients had hypertension, 30\% $(\mathrm{n}=16)$ had type 2 diabetes mellitus, 28\% ( $\mathrm{n}=15)$ patients had depression, $11 \%(\mathrm{n}=6)$ had COPD, 9\% ( $\mathrm{n}=5)$ had asthma. The patients had undergone either a laparoscopic sleeve gastrectomy (SG; $\mathrm{n}=31$ ), a Roux-en- $\mathrm{Y}$ gastric bypass (RYGB; $\mathrm{n}=20$ ), and in two patients the type of surgery was not documented, with or without a simultaneous hernia repair. There were no patient deaths in the operated population during the data collection period.

There were no significant differences between the surgical and non-surgical population with regards to 
Table 3 Comparison of pre-operative weight, BMI and weight loss following surgery in patients on/off CPAP

\begin{tabular}{lccc}
\hline Parameters & Surgical CPAP cohort & Surgical non-CPAP cohort & P value \\
\hline Weight $(\mathrm{kg})$ & $139.5(34.7) ; \mathrm{n}=25$ & $136.7(22.1) ; \mathrm{n}=28$ & 0.718 \\
BMI $\left(\mathrm{kg} / \mathrm{m}^{2}\right)$ & $49.7(10.4) ; \mathrm{n}=25$ & $49.1(5.9) ; \mathrm{n}=28$ & 0.794 \\
Weight loss $(\%)$ & $25.0(10.5) ; \mathrm{n}=18$ & $19.2(9.8) ; \mathrm{n}=20$ & 0.086 \\
\hline
\end{tabular}

$\mathrm{BMI}$, body mass index; CPAP, continuous positive airway pressure.

demographics, symptom scores or nocturnal pulse oximetry data. CPAP treatment had been commenced in 47\% (25:28) of this cohort, mean CPAP pressure was $13.1(2.2) \mathrm{cmH}_{2} \mathrm{O}$, nightly CPAP use was 4.2 (2.1) hours, and the ESS was 7.8 (5.0) points on treatment. The mean post-operative follow-up time was 375.3 (277.9) days. By then, patients had an average weight loss of $22.0 \%$ (10.5) of their body weight with no significant difference between the groups (Table 3). The average length of stay in hospital was 2.5 (1.2) days, there was no difference between patients on CPAP compared to those without [CPAP 2.6 (1.4) vs. no CPAP 2.4 (1.0) days, $\mathrm{P}=0.650]$.

Out of the 53 surgical patients, only 2 had any pre-, intra- or post-operative complications:

(I) A 63-year-old female patient with COPD, not receiving CPAP, suffered from an acute kidney injury (AKI) post laparoscopic sleeve gastrectomy. This patient was treated on a non-ICU hospital ward for 2 days before being discharged; the patient has not had any relevant medical complications in the 9 months since surgery;

(II) A 62-year-old female patient with COPD, extubated onto CPAP, suffered from hypercapnic (type 2) respiratory failure (HCRF) postlaparoscopic sleeve gastrectomy. This patient was admitted to ICU, and was treated in ICU for 2 days before being discharged. This patient has not suffered any relevant medical complications in the 4 months since surgery.

\section{Discussion}

OSA is the most common form of SDB and possibly the most prevalent chronic respiratory disorder (8). OSA is compounded by the fact that the morbidly obese population breathe at a reduced functional residual capacity, approaching the residual volume, which reduces lung compliance, increases the physical work of breathing and minimizes the pressure gradient between the alveolar and intrapleural pressure (trans-pulmonary pressure) (8) necessary to drive minute ventilation for continuous gas exchange $(6,9)$. However, increased levels of neural respiratory drive in obesity decrease with the onset of sleep (8). In addition, basal metabolic rate and oxygen demand are increased, leading to a rapid decline in arterial oxygen saturation when breathing is interrupted (6). Increased intra-abdominal pressure creates intrinsic positive end-expiratory pressure, particularly in supine posture (8), and functions as a limit to the inspiratory load $(6,9,15)$.

\section{Summary}

In a large cohort of bariatric surgery patients, sleepdisordered breathing is highly prevalent: $70 \%$ had some form of OSA, while $40 \%$ required peri-operative planning with CPAP therapy. Obese men had more severe OSA, while the majority of bariatric patients was female. Although only a minority of $13 \%$ had been operated during the follow-up period, only one patient ( $2 \%$ of the surgical cohort), who had COPD and was extubated onto CPAP, suffered post-operative respiratory complications. None of the other patients had any significant respiratory perioperative complications. There was no significant difference in length of hospital stay between operated patients with CPAP compared to the non-CPAP cohort. Pre-operative established CPAP therapy and/or extubation onto CPAP proved to deliver the same outcomes as in the patient cohort with no or only mild OSA; CPAP therapy has proven to deliver an effective method of controlling respiratory complications during and after surgery.

\section{Clinical significance}

Previous studies have found similar results with regards to rates of OSA in bariatric surgery cohorts, between $73 \%$ and $78 \%(12,13,16)$ of bariatric surgery patients have some form of sleep-disordered breathing, with $24 \%$ suffering from moderate to severe OSA and $11 \%$ having findings 
suggestive of combined OSA/OHS (12).

Sleep-disordered breathing is a significant risk factor for perioperative complications: a systematic review identified eight studies linking OSA to higher rates of hypercapnic respiratory failure (17). Patients with moderate to severe sleep-disordered breathing are at higher risk of mortality, sepsis and respiratory complications after elective coronary artery bypass-graft surgery (18). Patients with OSA and unidentified OHS had higher risks of postoperative respiratory failure, heart failure, prolonged intubation, and longer ICU and hospital length of stay than patients with non-hypercapnic OSA (19).

Obese patients with OSA present a challenge to anaesthetists, with a significantly higher risk of difficult mask ventilation and laryngoscopy (20), as well as requiring more intubation attempts (21). Meta-analyses have established that patients with OSA are more than twice as likely to suffer peri-operative respiratory complications compared to patients without OSA $(22,23)$. Many other types of surgery carry an inherently increased risk of respiratory complications, even in patients with no prior risk factors (11). Screening for OSA may thus be applicable to these types of surgery as well as bariatric surgery.

Besides a difficult airway with high Mallampati score, COPD is one of the most commonly identifiable causes of post-operative pulmonary complications. OSA may be prevalent in $13.8 \%$ of COPD patients and significantly compounds peri-operative risk (24). Pre-operative screening for OSA (and treatment with CPAP if necessary) in pre-surgical COPD patients may be of clinical importance. However, $23 \%$ of patients with combined COPD and OSA may require bilevel positive airway pressure (BiPAP) for associated hypoventilation with hypercapnic respiratory failure (24). Hypercapnic (type 2) respiratory failure increases mortality (25), particularly in patients with COPD, even in those treated on a respiratory ITU (26). The COPD patient who suffered from a postoperative HCRF in our cohort may therefore have required non-invasive ventilation instead of CPAP and as such may not represent a lack of efficacy in peri-operative CPAP treatment.

CPAP is a routine and beneficial treatment for moderate to severe OSA (27) and has been recorded as an effective method of reducing peri-operative respiratory complications compared to patients with undiagnosed OSA without receiving CPAP $(11,28)$. Although CPAP does reduce symptoms relating to apnoea, such as abnormalities in night-time oxygen levels and excessive daytime sleepiness, it has also positive effects on memory and cognition $(29,30)$, improves quality of life, reduces blood pressure (31) and improves cardiac outcomes (32). However, despite patients experiencing relief of sleep-related symptoms, compliance to CPAP treatment remains suboptimal, with as many as half of the patients not sufficiently adhering to treatment at 1 year (17). Indeed, our study found that patients only used their prescribed CPAP during two out of every three nights, on average.

The similar length of stay between the groups with and without OSA highlights the effectiveness of CPAPpatients prescribed appropriate CPAP had outcomes and hospital stays comparable to patients who did not require it at all. This demonstrates that CPAP is effective in managing the respiratory needs of bariatric patients and those with OSA.

The ESS is the most commonly used questionnaire in Sleep Med (13), but it does not differentiate between various causes of excessive daytime sleepiness (EDS) (33), or correlate with objective measures of EDS (9) and its applicability to obese patients is controversial (34). For these reasons, the STOP-Bang questionnaire has taken precedence for OSA screening in obese patients in the pre-operative clinics (20). The STOP-Bang questionnaire is based on snoring, daytime tiredness, observed apnoeic episodes, BMI over $35 \mathrm{~kg} / \mathrm{m}^{2}$, advanced age, male gender, a history of hypertension and neck circumference (35). These criteria are highly sensitive (94-96\%) in the diagnosis of moderate-severe OSA (36).

\section{Limitations}

Patients studied were selected solely based on high-risk STOP-Bang scores, thus patients with lower scores were not followed. Future research should attempt to include all surgical patients to determine the presence (or absence) of any peri-operative respiratory complications in those not screened via the sleep service. Additionally, although highly sensitive, the STOP-Bang score is not a measure of OSA, and patients with actual OSA may not have a high STOPBang score, excluding them from the cohort despite their suitability for treatment.

Furthermore, all patients with moderate-severe OSA were treated with CPAP, and we cannot claim that there was an increased risk of complications if these patients had remained untreated. However, the fact that overall risks were low and comparable between groups is reassuring in that the proposed management plan is sufficient. In 
the context of a randomised controlled clinical trial, the use of sham-CPAP, an effective placebo for CPAP (37), if ethically acceptable, would provide more evidence about the effectiveness of peri-operative CPAP. Hip circumference measurements would have been of interest for assessing insulin resistance. HAD scores for surgical patients would have also been useful to assess the psychological profile of this cohort. However, the surgical cohort matched the non-surgery cohort, and it is therefore likely that the HAD scores could be assumed to be similar. Additionally, although CPAP is effective in OSA, BiPAP may be more suitable for patients with combined OSA and COPD.

Lastly, this study focused on peri-operative outcomes in bariatric/abdominal surgery, but pre-operative OSA screening and subsequent CPAP treatment may be applicable to other forms of (respiratory) high-risk surgery as well.

\section{Conclusions}

Bariatric patients screened pre-operatively for OSA and treated with CPAP, according to guidelines, suffer few respiratory complications. Pre-operative screening for OSA and peri-operative treatment with CPAP in morbidly obese patients is an effective method of reducing respiratory disease-related morbidity and mortality in the bariatric population.

\section{Acknowledgements}

Dr. Steier's contribution to this project was supported by the National Institute for Health Research (NIHR) Biomedical Research Centre based at Guy's and St. Thomas' NHS Foundation Trust and King's College London, who part funded his salary. The views expressed are those of the authors and not necessarily those of the NHS, the NIHR or the Department of Health.

\section{Footnote}

Conflicts of Interest: The authors have no conflicts of interest to declare.

Ethical Statement: The study was approved by the local clinical governance board (GSTT-2017-6952).

\section{References}

1. McPherson K, Marsh T, Brown M. Tackling obesities: future choices-modelling future trends in obesity and the impact on health. UK Government Office for Science. 2nd ed. Available online: www.foresight.gov.uk

2. Health and Social Care Information Centre. Statistics on Obesity, Physical Activity and Diet. Leeds, England: Health and Social Care Information Centre, 2013.

3. Zammit C, Liddicoat H, Moonsie I, et al. Obesity and respiratory diseases. Int J Gen Med 2010;3:335-43.

4. Mutter TC, Chateau D, Moffatt M, et al. A matched cohort study of postoperative outcomes in obstructive sleep apnea: could preoperative diagnosis and treatment prevent complications? Anesthesiology 2014;121:707-18.

5. Steier J, Martin A, Harris J, et al. Predicted relative prevalence estimates for obstructive sleep apnoea and the associated healthcare provision across the UK. Thorax 2014;69:390-2.

6. Members of the Working Party, Nightingale CE, Margarson MP, et al. Peri-operative management of the obese surgical patient 2015: Association of Anaesthetists of Great Britain and Ireland Society for Obesity and Bariatric Anaesthesia. Anaesthesia 2015;70:859-76.

7. Pengo MF, Drakatos P, Kosky C, et al. Nocturnal pulse rate and symptomatic response in patients with obstructive sleep apnoea treated with continuous positive airway pressure for one year. J Thorac Dis 2014;6:598-605.

8. Steier J, Jolley CJ, Seymour J, et al. Neural respiratory drive in obesity. Thorax 2009;64:719-25.

9. Steier J, Kent B. Assessment and treatment of sleepdisordered breathing in the morbidly obese surgical patient. Cambridge University Press, 2017. [Epub ahead of print].

10. Smetana GW. Preoperative pulmonary evaluation. N Engl J Med 1999;340;937-44.

11. Qaseem A, Snow V, Fitterman N, et al. Risk assessment for and strategies to reduce perioperative pulmonary complications for patients undergoing noncardiothoracic surgery: a guideline from the American College of Physicians. Ann Intern Med 2006;144:575-80.

12. Reed K, Pengo MF, Steier J. Screening for sleepdisordered breathing in a bariatric population. J Thorac Dis 2016;8:268-75.

13. Johns MW. A new method for measuring daytime sleepiness: the Epworth sleepiness scale. Sleep 1991;14 540-5.

14. Bjelland I, Dahl AA, Haug T, et al. The validity of the Hospital Anxiety and Depression Scale: an updated literature review. J Psychosom Res 2002;52:69-77.

15. Steier J, Jolley CJ, Seymour J, et al. Increased load on 
the respiratory muscles in obstructive sleep apnea. Respir Physiol Neurobiol 2010;171:54-60.

16. Lopez PP, Stefan B, Schulman CI, et al. Prevalence of sleep apnea in morbidly obese patients who presented for weight loss surgery evaluation: more evidence for routine screening for obstructive sleep apnea before weight loss surgery. Am Surg 2008;74:834-8.

17. Opperer M, Cozowicz C, Bugada D, et al. Does Obstructive Sleep Apnea Influence Perioperative Outcome? A Qualitative Systematic Review for the Society of Anesthesia and Sleep Med Task Force on Preoperative Preparation of Patients with Sleep-Disordered Breathing. Anesth Analg 2016;122:1321-34.

18. Rupprecht S, Schultze T, Nachtmann A, et al. Impact of sleep disordered breathing on short-term post-operative outcome after elective coronary artery bypass graft surgery: a prospective observational study. Eur Respir J 2017;49. pii1601486.

19. Chung F, Nagappa M, Singh M, et al. Contemporary Reviews in Sleep Med CPAP in the Perioperative Setting: Evidence of Support. Chest 2016;149:586-97.

20. Kheterpal S, Healy D, Aziz MF, et al. Incidence, predictors, and outcome of difficult mask ventilation combined with difficult laryngoscopy: a report from the multicenter perioperative outcomes group. Anesthesiology 2013;119:1360-9.

21. Stierer TL, Wright C, George A, et al. Risk assessment of obstructive sleep apnea in a population of patients undergoing ambulatory surgery. J Clin Sleep Med 2010;6:467-72.

22. Kaw R, Chung F, Pasupuleti V, et al. Meta-analysis of the association between obstructive sleep apnoea and postoperative outcome. Br J Anaesth 2012;109:897-906.

23. Hai F, Porhomayon J, Vermont L, et al. Postoperative complications in patients with obstructive sleep apnea: a meta-analysis. J Clin Anesth 2014;26:591-600.

24. Kuklisova Z, Tkacova R, Joppa P, et al. Severity of nocturnal hypoxia and daytime hypercapnia predicts CPAP failure in patients with COPD and obstructive sleep apnea overlap syndrome. Sleep Med 2017;30:139-45.

25. Hukins C, Wong M, Murphy M, et al. Management of hypoxaemic respiratory failure in a Respiratory Highdependency Unit. Intern Med J 2017;47:784-92.

26. Khilnani GC, Banga A, Sharma SK. Predictors of mortality of patients with acute respiratory failure secondary to chronic obstructive pulmonary disease admitted to an intensive care unit: a one year study. BMC Pulm Med 2004;4:12.
27. Giles TL, Lasserson TJ, Smith BJ, et al. Continuous positive airways pressure for obstructive sleep apnoea in adults. Cochrane Database Syst Rev 2006;(1):CD001106.

28. Gami AS, Howard DE, Olson EJ, et al. Day-night pattern of sudden death in obstructive sleep apnea. $\mathrm{N}$ Engl J Med 2005;352:1206-14.

29. Ferini-Strambi L, Baietto C, Di Gioia MR, et al. Cognitive dysfunction in patients with obstructive sleep apnea (OSA): partial reversibility after continuous positive airway pressure (CPAP). Brain Res Bull 2003;61:87-92.

30. Dalmases $M$, Solé-Padullés $C$, Torres $M$, et al. Effect of CPAP on Cognition, Brain Function, and Structure Among Elderly Patients With OSA: A Randomized Pilot Study. Chest 2015;148:1214-23.

31. McNicholas WT, Bonsigore MR, Management Committee of EU COST ACTION B26. Sleep apnoea as an independent risk factor for cardiovascular disease: current evidence, basic mechanisms and research priorities. Eur Respir J 2007;29:156-78.

32. Doherty LS, Kiely JL, Swan V, et al. Long-term effects of nasal continuous positive airway pressure therapy on cardiovascular outcomes in sleep apnea syndrome. Chest 2005; 127:2076-84.

33. Slater G, Steier J. Excessive daytime sleepiness in sleep disorders. J Thorac Dis 2012;4:608-16.

34. Slater G, Pengo MF, Kosky C, et al. Obesity as an independent predictor of subjective excessive daytime sleepiness. Respir Med 2013;107:305-9.

35. Chung F, Yegneswaran B, Liao P, et al. STOP questionnaire: a tool to screen patients for obstructive sleep apnea. Anesthesiology 2008;108:812-21.

36. Nagappa M, Liao P, Wong J, et al. Validation of the STOP-Bang Questionnaire as a Screening Tool for Obstructive Sleep Apnea among Different Populations: A Systematic Review and Meta-Analysis. PLoS One 2015;10:e0143697.

37. Rodway GW, Weaver TE, Mancini C, et al. Evaluation of sham-CPAP as a placebo in CPAP intervention studies. Sleep 2010;33:260-6.

Cite this article as: Meurgey JH, Brown R, WoroszylChrusciel A, Steier J. Peri-operative treatment of sleepdisordered breathing and outcomes in bariatric patients. J Thorac Dis 2018;10(Suppl 1):S144-S152. doi: 10.21037/ jtd.2017.10.11 